Cahiers de recherches médiévales

\title{
Compléter la Table Ronde
}

Le lignage de Guiron vu par les armoriaux arthuriens

\section{Richard Trachsler}

\section{(2) OpenEdition \\ Journals}

Édition électronique

URL : https://journals.openedition.org/crm/2659

DOI : $10.4000 / \mathrm{crm} .2659$

ISSN : 1955-2424

Éditeur

Honoré Champion

\section{Édition imprimée}

Date de publication : 15 décembre 2007

Pagination : 101-114

ISSN : 1272-9752

\section{Référence électronique}

Richard Trachsler, «Compléter la Table Ronde », Cahiers de recherches médiévales [En ligne], 14 | 2007, mis en ligne le 15 décembre 2010, consulté le 15 décembre 2022. URL : http://

journals.openedition.org/crm/2659; DOI : https://doi.org/10.4000/crm.2659 


\section{酷M}

\section{Compléter la Table Ronde. Le lignage de Guiron vu par les armoriaux arthuriens}

Un armorial est, pris à la lettre, un «livre des armes », c'est-à-dire que l'on y consigne les armoiries des chevaliers d'une famille, d'une région, d'un pays ou tout simplement de ceux qui ont été présents à un événement particulier. À l'opposé d'un tel armorial «occasionnel», comme on dit, se situent l'armorial "général», qui recense les armoiries de toute la noblesse occidentale, et l'armorial « universel» qui comporte en outre des personnages préhéraldiques, comme Charlemagne, les héros bibliques ou le prêtre Jean ${ }^{1}$. Les armoriaux arthuriens, dont il sera ici question, sont de la même veine dans la mesure où il y est question de personnages non historiques $^{2}$ : il s'agit en effet de répertoires qui donnent la liste de l'ensemble des chevaliers de la Table Ronde, avec leur nom et leur écu, le plus souvent dessiné, parfois aussi blasonné, occasionnellement accompagné de renseignements concernant les supports, le cimier et la devise du chevalier ${ }^{3}$. À côté de cette première forme de l'armorial arthurien, il en existe une seconde, qui ajoute, à ces informations héraldiques, une petite description de chaque personnage, voire son portrait.

Dans les manuscrits qui comportent des descriptions, le tout est précédé d'une rubrique liminaire destinée à conférer aux différentes notices une sorte d'unité de temps et de lieu :

Ce sont les noms, armes et blasons des chevaliers compaignons de la Table Ronde au temps que ilz jurerent la queste du Sainct Graal a Camaloth le jour de la

\footnotetext{
${ }^{1}$ M. Pastoureau, Traité d'Héraldique, Paris, Picard, 1979 (Grands Manuels Picard), p. 27-28 ainsi que son fascicule Les Armoiries, Turnhout, Brepols, 1976 (Typologie des sources du Moyen Âge occidental, fasc. 20).

${ }^{2}$ L'ouvrage de base reste le livre de M. Pastoureau, Armorial des Chevaliers de la Table Ronde, Paris, Le Léopard d'Or, 1983. L'imprimé réalisé en 1590 par Benoît Rigaud est devenue facilement accessible: La Devise des armes des chevaliers de la table ronde, lesquels estoyent du tres-renommé \& vertueux Artus, Roy de la grand bretaigne, Lyon, Benoist Rigaud, 1590, Réimpression: Ivry, Phénix Éditions, 2000. Il ne s'agit pas, comme l'indique la quatrième de couverture, d'un album à colorier, mais d'un armorial arthurien, comme l'avait déjà vu C. E. Pickford, L'Évolution du roman arthurien en prose vers la fin du Moyen Âge d'après le manuscrit 112 du fonds français de la Bibliothèque Nationale, Paris, Nizet, 1960, p. 256. Pour une présentation de la tradition manuscrite de ces armoriaux ainsi qu'un choix de trente-trois biographies, voir mon livre Clôtures du Cycle Arthurien. Étude et Textes, Genève, Droz, 1996 (Publications Romanes et Françaises 225).

${ }^{3}$ Pour se faire une idée de l'apparence d'un tel manuscrit, voir L. Jefferson, «Tournaments, Heraldry and the Knights of the Round Table: A Fifteenth-Century Armorial with Two Accompanying Texts», Arthurian Literature, 14, 1996, p. 69-157. Elle reproduit un document publié jadis par le comte de Blangy et aujourd'hui dans une collection privée. Grâce à la générosité du collectionneur anonyme, des reproductions en couleurs ont pu être jointes à la publication.
}

Cahiers de Recherches Médiévales, 14, 2007 

premierement. ${ }^{4}$

C'est, pour ainsi dire, le degré zéro d'un récit-cadre, qui vise à présenter l'armorial comme la photo instantanée d'un moment précis de l'histoire arthurienne connu de tous. De la sorte, les informations diverses et hétéroclites concernant les biographies, écus et noms de plus de cent cinquante chevaliers sont insérées dans la fiction arthurienne au lieu de se présenter simplement comme une sorte de fichier regroupant les membres de la Tables Ronde.

De tous ces éléments, ce sont naturellement les descriptions elles-mêmes qui retiennent l'attention de l'historien de la littérature bien que l'invention des écus, qui s'appuie en général sur le simple nom propre, soit elle aussi riche en renseignements sur la sensibilité médiévale. La longueur de ces descriptions oscille entre quelques lignes pour un chevalier peu connu, et plusieurs feuillets pour les personnages majeurs comme Arthur, Lancelot, Galaad, Perceval et Tristan. Quant au contenu des notices, il est lui aussi fonction de la célébrité des personnages, car si l'auteur est à peu près libre d'inventer une apparence physique, des origines, des habitudes vestimentaires ou gastronomiques pour un chevalier mineur, il est davantage contraint quand il s'agit des grands noms de la littérature arthurienne, où il doit respecter les données léguées par la tradition.

Voici, à titre d'exemple, la notice de Dodinel le Sauvage, chevalier de second plan qui apparaît régulièrement dans le décor des romans arthuriens depuis le Lancelot en prose ${ }^{5}$.

Cy devise de Dodinel le Sauvage

Dodinel le Sauvage estoit d'Escosse et estoit grant homme aucques gras, le visage eust ung peu trop couloré et moult estoit gros de tous ses membres et n'eust guieres cheveulx. Fort homme estoit a desmesure et si bon chevalier que c'estoit merveilles, moult estoit a craindre quant il se courroussoit et congnoissoit bien le fait de la guerre. Tousjours queroit avantures estranges. Moult mangeoit et buvoit fort. Loyal homme fut a merveilles et le roy Artus se fioit fort a luy. Et portoit en ses armes d'argent a une aigle d'asur membree d'asur. ${ }^{6}$

La notice respecte les données traditionnelles, puisqu'elle arrive, à partir de la «sauvagerie » du nom propre, au caractère boulimique et colérique du personnage.

\footnotetext{
${ }^{4}$ Manuscrit de PARIS, BNF, f. fr. 12597 , fol. $1^{\mathrm{r}}$.

${ }^{5}$ Pour les détails, au demeurant fort complexes, voir G. D. West, An Index of Proper Names in Arthurian Verse Romances, 1150-1300, Toronto, Toronto University Press, 1969 (University of Toronto Romance Series 15), p. 50-51 et Id., An Index of Proper Names in Arthurian Prose Romances, Toronto, Toronto University Press, 1978 (University of Toronto Romance Series 35), p. 95-96. Pour une présentation du matériel, on peut consulter les études de G. Huet, «Deux Personnages arturiens [!]», Romania, 43, 1914, p. 96-102 et d'E. Brugger, "Bliocadran, the Father of Perceval», Mediaeval Studies in Memory of Gertrude Schoepperle-Loomis, Paris-New York, Champion-Columbia University Press, 1927, p. 14774, en particulier p. 166-71.

${ }^{6}$ Manuscrit de PARIS, BNF 12597, fol. $37^{\mathrm{r}}$. Le manuscrit porte ung aigle.
} 
En même temps, elle y ajoute aussi quelque chose: en l'occurrence, il s'agit du détail «qui ne s'invente pas» concernant sa calvitie, qui, sauf erreur, ne se trouve pas dans les sources, pas plus que les renseignements concernant le teint et la corpulence du chevalier.

La description de Dodinel est très représentative de la façon de travailler de l'auteur de cet armorial puisqu'elle montre bien le louvoiement entre l'invention, qui perce dans les détails, et le respect de la tradition pour ce qui concerne les grandes lignes. Un autre trait qui parcourt une grande partie des notices est le lien qui rattache le chevalier à la cour d'Arthur, élément qui confère à toutes ces notices une certaine unité et les scelle dans la grande fratrie élitiste qu'est la communauté de la Table Ronde.

Dans le cas de la "génération des pères", c'est-à-dire les héros de la génération d'avant Arthur, qui feront l'objet de ces pages, les choses se présentent de façon différente par rapport au reste des chevaliers, car ces chevaliers antérieurs à l'ère de la quête du Graal, où a été prise la «photo» que conservent les armoriaux, forment un bloc à part, quantitativement plus réduit que leurs descendants et rajouté après-coup. Il peut donc être intéressant d'examiner les notices biographiques des Guiron, Meliadus, Danaain et autres pour voir ce qu'elles apportent par rapport à celles des « jeunes ».

La présence de la génération des pères est bien une adjonction par rapport au «tronc» de l'armorial, dont le noyau est très vraisemblablement la liste des quêteurs telle qu'elle est donnée dans le Tristan en prose, lors de la Pentecôte du Graal'. L'ordre dans lequel les chevaliers apparaissent, au moins au début et à la fin, est en effet assez conforme à cette liste, au demeurant plutôt fluctuante dans la tradition manuscrite $^{8}$. En tout état de cause, cette liste dépasse à peine les cent noms et l'auteur des armoriaux a donc dû l'étoffer par des personnages créés ad hoc, afin d'atteindre le chiffre de cent cinquante, nombre canonique des chevaliers admis à la Table Ronde dans les romans en prose ${ }^{9}$. Les manuscrits comportant des armoriaux

\footnotetext{
${ }^{7}$ Le rapprochement a été fait par Pickford, L'Évolution, op. cit., p. 256.

${ }^{8}$ Pour le passage en question cf. Tristan, vol. VI, éd. E. Baumgartner \& M. Szkilnik, Genève, Droz, 1993 (TLF 437), p. 273-74. C.-A. van Coolput, «Aventures querant et le sens du monde». Aspects de la réception des premiers romans du Graal cycliques dans le «Tristan en Prose», Leuven, Leuven University Press, 1986 (Mediaevalia Lovaniensia 13), p. 252-53, a édité le passage d'après le BNF f. fr. 336. Cf. aussi E. Löseth, Le roman en prose de Tristan, le roman de Palamède et la compilation de Rusticien de Pise. Analyse critique d'après les manuscrits de Paris, Paris, Champion, 1890 (Bibl. de l'École des Hautes Études LXXXII) [réimpr. Genève, Slatkine, 1974], § 395a, qui donne d'autres variantes de cette liste fatalement instable dans la tradition manuscrite.

${ }^{9}$ Sur la Table Ronde, voir H. Eberlein-Westhues, « König Arthurs «Table Ronde». Studien zur Geschichte eines literarischen Herrschaftszeichens", Der altfranzösische Prosaroman. Funktion, Funktionswandel und Ideologie am Beispiel des «Roman de Tristan en prose». Kolloquium Würzburg, 1977, éd. par E. Ruhe et R. Schwaderer, München, Fink, 1979 (Beiträge zur romanischen Philologie des Mittelalters XII), p. 184-263. À propos des créations de personnages, voir mes observations dans Disjointures-Conjointures. Étude sur
} 
sans biographies donnent d'ailleurs exactement cent cinquante écus, ce qui prouve que l'idée de départ était bien de capter le reflet de la Table Ronde au moment de la quête du saint Vaisseau, plus exactement lors de la Pentecôte du Graal, quand la gloire d'Arthur était à son zénith ${ }^{10}$.

C'est en effet uniquement dans les armoriaux avec descriptions que l'on trouve, antéposée aux quêteurs du Graal, une trentaine de chevaliers appartenant, en principe, à l'époque d'avant, celle des pères. Dans le détail, il s'agit de Guiron [1] et de son lignage, représenté par Séguran le Brun [2] et Gallinan le Blanc [3], fils de Guiron; Meliadus [4], le père de Tristan, Ban de Benoïc [5], père de Lancelot, et Boort de Gaunes [6], père du héros du Graal homonyme. Vient ensuite une série de rois, avec Karadoc Briébras [7], Lac [8], le roi d'Estrangorre, Brunor le roi, surnommé le Bon Chevalier sans peur [9], Clarés [10], Urien [11], Loth[13], Leodegan [14], Rion [13], Yder [15], Pelinor [16], Baudemagu [17], Gallegatin de Norgalles [18], Aguisant d'Écosse [19], Malaquin [20], Le Morholt d'Irlande ${ }^{11}$ [21] et Pharamon de Gaules [22]. Le dernier groupe de chevaliers est constitué de personnages qui jouissent d'une certaine réputation dans le cycle de la Post-Vulgate ou dans l'ensemble Guiron-Meliadus: Lamorat de Listenois senior [23], Danain le Roux [24], grand ami de Guiron, Ariolan de Sessoigne [25], Ballaan [26] et son frère Baalain [27], Galehaut [28], Blanor de Gaunes [29], le Bon Chevalier de Norgalles [30], Hervi de Rivel [31] et, finalement, Lamorat de Listenois iunior [32].

Pour être tout à fait précis, il faudrait dire que ces chevaliers «anciens» ne sont pas simplement antéposés, mais intercalés entre la notice consacrée à Arthur lui-même et la série des quêteurs, qui débute, dans les armoriaux comme dans le Tristan en prose, par la mention de Galaad, Perceval, Lancelot, Boort et se clôt, toujours comme la liste du Tristan, sur Felix ou Philip le Quérant, personnage autrement inconnu. C'est une preuve supplémentaire de la dépendance étroite des armoriaux de cette liste. Très vraisemblablement, quelqu'un a interpolé ces noms dans la séquence originelle fournie par la scène de la Pentecôte du Graal, ce qui a entraîné une sorte de bipartition du livre. L'auteur en était bien conscient. Au moment de commencer son texte, il parle d'une premiere partie, et à la transition, entre la présentation des ancêtres et celle des quêteurs du Graal, il insère une nouvelle rubrique liminaire.

C'est la seconde partie de la devise des meurs et condicions des chevaliers de la Table Ronde et la façon de leurs personnes qui furent faictz chevaliers ou temps du roy Artus. Et les descripvay en la maniere qu'ilz estoient assis a la Table Ronde quant la grant conqueste du Sainct Graal commença. Et bien est vray que tous ceulx la qui estoient roys, que j'ay devisé en la premiere partie, tant pour honneur de leur

l'interférence des matières narratives dans la littérature française du Moyen Âge, TübingenBasel, A. Francke Verlag, 2000 (Romanica Helvetica 120), p. 53-59.

${ }^{10}$ Pour être tout à fait précis, il faudrait dire que ces manuscrits comportent parfois un $151^{\mathrm{e}}$ écu, celui de Palamède, rajouté à la fin, puisque le vaillant sarrasin n'a pas participé à la Quête et n'a accepté de se convertir et de siéger à la Table Ronde qu'après la mort de Tristan.

${ }^{11}$ Dans les manuscrits, le nom est souvent estropié et transformé en Lamora(n)t d'Irlande. 
royaulté comme pource que la plus partie estoient chevaliers anciens et du temps du roy Uterpandragon. ${ }^{12}$

Cette rubrique s'achève sur une phrase étrange, assez répandue dans la tradition manuscrite: Et ores commenceray ma premiere partie, où premiere est sans aucun doute une faute pour seconde, puisque la première partie, celle qui est consacrée à la génération ancienne, vient justement de se clore ${ }^{13}$. Cette leçon, qui doit provenir du modèle, c'est-à-dire d'un armorial à cent cinquante chevaliers, traduit bien la perturbation que subit l'ordre du livre à cause de la présence de ces intrus. Ces «anciens", impossibles à caser dans le cadre temporel prévu pour la photo de groupe à la Pentecôte du Graal, obligent en effet à éclater ce cadre spatiotemporel commode pour en proposer un second, plus en amont. Du coup, la concurrence naît: il y a deux plans, deux décors, deux photos de groupe et chaque personnage n'a droit qu'à une prise, alors que les candidats à cheval sur les deux époques et qui pourraient donc prétendre à figurer sur les deux photos existent bel et bien.

En effet, certains anciens ont beau être des anciens et avoir vécu les temps glorieux de la jeunesse de l'aetas arturiana, ils n'en sont pas pour autant trop anciens pour participer à la Quête du Graal. Mais ils ont tous été comptabilisés parmi la génération des pères, lors de leur première apparition. Il en va ainsi de Loth, Baudemagu, Yder, Rion, Kardados et le roi de Claré, ainsi que de Galegantin, Aguisant et Malaquin, sans exception des «ancêtres » qui participent aussi à la Quête du Graal. Comme il ne pouvait évidemment pas être question de donner deux fois, en l'espace de quelques feuillets, la description d'un même personnage, on lit, dans l'énumération des quêteurs du Graal, des renvois du type :

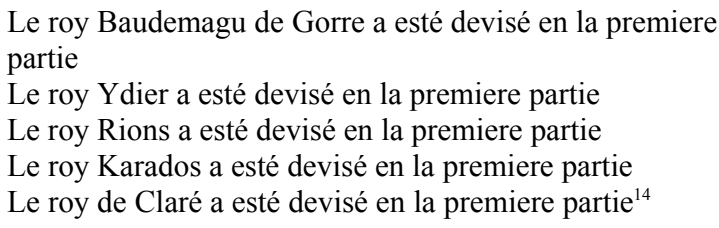

Venant juste après Galaad, Perceval, Lancelot, Boort, Gauvain, Tristan, Lionel et Helyas le Blanc, ce groupe de quêteurs - de Baudemagus au roi de Claré -

\footnotetext{
${ }^{12}$ Manuscrit de PARIS, BNF f. fr. 12597, fol. $19^{\mathrm{r}}$.

${ }^{13}$ Je n'ai trouvé nulle part la leçon attendue, seconde. Premiere se lit, outre dans le fr. 12597, aussi dans le fr. 1437, qui fait pourtant tout pour signaler qu'il commence la seconde partie : « $\left[41^{\mathrm{v}}\right]$ C'est la seconde partie de la devise des meurs et condicions des chevaliers de la Table Ronde et la façon de leur maniere de faire et vivre qui furent faictz chevaliers au temps du roy Artus. $\left[42^{\mathrm{r}}\right]$ Et les descripv[er]ay en la maniere qu'ilz estoient assis a la Table Ronde quant la grant conqueste du Sainct Graal commença. Et bien est vray que tous ceulx la qui estoient roys que j'ay devisé en la premiere partie, tant pour l'honneur de leur royaulté comme pour ce la plus partie estoient chevaliers anciens et du temps du roy Uterpandragon. Et ores commenceray ma premiere partie. »

${ }^{14}$ Manuscrit de PARIS, BNF f. fr. 12597, fol. $28^{\mathrm{r}}$.
} 
se retrouve exactement à la même position dans la liste des quêteurs contenue dans le Tristan en prose ${ }^{15}$.

On voit que l'adjonction de la premiere partie consacrée à ces old timers a brisé la belle unité de temps et de lieu qu'apportait la Pentecôte du Graal, modifiant par la même occasion la structure du livre et entraînant des perturbations dont la tradition textuelle accuse les traces. Il vaut donc la peine de regarder d'un peu plus près les personnages introduits en ouverture du livre. On se limitera surtout à quelques protagonistes appartenant au lignage de Guiron le Courtois, dont on présentera et étudiera les descriptions.

Ce qui frappe de prime abord, quand on parcourt la liste des trente-deux personnages surnuméraires par rapport à la scène du Graal, c'est la grande variété des sources dont ils semblent provenir: si certains renvoient indubitablement à l'univers du Guiron-Meliadus, d'autres évoquent l'univers du Tristan et du Lancelot en prose, voire du cycle de la Post-Vulgate. La «contamination» des deux univers du Guiron-Meliadus, avec le cycle de la Post-Vulgate, est tellement visible qu'on se demande s'il n'y a pas eu une source particulière. En l'occurrence, il pourrait s'agir d'un manuscrit dont LONDON, British Library, Additional 36673 conserve le reflet. $\mathrm{Ce}$ manuscrit, qui date certes $\mathrm{du} \mathrm{XVI}{ }^{\mathrm{e}}$ siècle, donne une version très marginale au sein de la tradition textuelle du Guiron, mais qui se caractérise par une tendance à la compilation en même temps qu'à l'anthologie. Le tout est cependant maintenu par un cadre temporel suffisamment rigide pour conférer aux différents épisodes une tendance centripète certaine, assez voisine, au fond, de celle qui caractérise les armoriaux arthuriens.

Quelle que soit la provenance des récits, des personnages et de leurs aventures, leur présence dans un seul et unique livre est justifiée par l'argument imparable fournie par la chronologie: dans l'armorial, c'est la Pentecôte du Graal qui fixe les repères, délimitant, en l'occurrence, une seule journée; pour l'Additional 36673, c'est un segment temporel plus large qui est rempli. Le sommaire permet de s'en faire une idée assez représentative :

Cy commence le livre de missire Guiron le courtois du Boys Verdoyant et sera divisé en trois parties. La premiere sera au commencement du lignaige des Bruns et finera a la mort du roy Pelinor de Listenoys; la seconde quant Guiron saillit de la prison Luces le geant et finira a l'enprisonnement des bons chevaliers; la tierce a la naissance Lancelot et Tristan et finera a la mort de Galinant le Blanc. ${ }^{16}$

Dans ce contexte guironien se trouve en effet intercalée une histoire importante tirée du cycle de la Post-Vulgate, celle de la mort de Loth et de Pellinor. Le double homicide des deux chefs de file se trouve à l'origine de l'animosité entre

\footnotetext{
${ }^{15}$ Tristan, vol. VI, éd. Baumgartner \& Szkilnik, § 112, II. 36-41.

${ }^{16}$ London, British Library, Additional 36673, fol. $2^{\mathrm{r}}$, cité d'après R. Lathuillère, Guiron le Courtois. Étude de la tradition manuscrite et analyse critique, Genève, Droz, 1966 (Publications Romanes et Françaises LXXXVI), p. 49, où l'on trouvera aussi une description succincte du manuscrit.
} 
les deux lignages telle que la narrent le Tristan en prose ou la Post-Vulgate ${ }^{17}$. Chronologiquement, la mort de Pellinor et de Loth, dans le cycle de la Post-Vulgate, se situe bien à la même époque que les exploits de Guiron et rien ne s'oppose donc à une fusion des deux univers littéraires par télescopage, comme l'opère l'Additional 36673.

L'idée d'une coupe synchronique à travers les années fondatrices du royaume arthurien, coupe qui traverse et mélange tous les textes romanesques, n'est donc pas propre à l'auteur de l'armorial, mais se trouve aussi dans le manuscrit londonien, dont le modèle peut très bien être antérieur à la composition des notices des armoriaux arthuriens: de toute évidence, l'Additional 36673 est le résultat d'un assemblage de plusieurs rédactions, affecté de contradictions internes héritées de différentes sources. Il est donc clair que la matière du manuscrit londonien a circulé avant le $\mathrm{XVI}^{\mathrm{e}}$ siècle et que l'auteur des armoriaux a pu s'en inspirer. En effet, il existe aussi une rencontre textuelle entre le LONDON, British Library, Additional 36673 et la notice consacrée à Guiron dans les armoriaux, rencontre qui accrédite l'hypothèse d'une dette de l'auteur des armoriaux à l'égard d'une rédaction spécifique du Guiron dont le manuscrit londonien conserve la trace ${ }^{18}$.

Cy aprés parle du lignage des Bruns et de la ou ilz vindrent. Et premierement devise de la façon et meurs de Guiron le Courtoys.

Ainsi dit ores le compte que les geans occupoient la terre d'Escosse et vindrent deux chevaliers moult preudommes de leurs corps, dont l'un s'appelloit Albrun et l'autre Dombart, et firent tant par leur puissance qu'il rebouterent les geantz hors dudit royaume et gaignerent une isle de mer qu'ilz appellerent le Val de Bruns. Et de ceulx si saillirent tous les chevaliers que je nommeray cy dessoubz et si grant loz accrurent de chevalerie que en tout le monde n'y avoit lignaige si redoubté comme estoit le leur. Mais ne vous les nommeray pas tous fors les aucuns qui furent les plus vaillans. Il en issit Phebus le Fort, qui soy .xi.eme desconfit cincq roy pour ung et morut pour la belle damoyselle $\left[3 \mathrm{r}^{\circ}\right]$ de Norhombellande et fut ensevely en une cave ou Bruns sans Pitié entra une foys. Mais de celuy ne vous parleray je plus. Aprés fut de celuy lignaige Hector le Brun, Brunor ${ }^{19}$ le Brun, Galeoth le Brun. Ceulx cy furent si merveilleux chevaliers que tout le siecle en parlera a jamais. Qui de ceulx cy vouldra savoir la verité sy aille veoir la grant ystoire de messire Guiron le Courtois du Bois Verdoyant, car celuy en parle au long et a la verité. Mais de messire Guiron le Courtoys vous veuil dire et deviser la façon et meurs et quelles armes il portoit. Il estoit mout grant de corps et presque geant, de cheveulx n'eust pas moult grant planté, mais estoient de belle coulleur come blons. Le visaige eust fort blanc et si

\footnotetext{
${ }^{17}$ De ce fait, le manuscrit a fait l'objet d'une étude de F. Bogdanow, «Pellinor's Death in the Suite du Merlin and the Palamedes, MS. Brit. Mus. Add. $36673 »$, Medium Aevum, 29 (1960), p. 1-9. L'érudite anglaise ne se prononce pas sur le rôle du scribe de l'Add. 36673, qui pourrait, d'après elle, avoir inventé l'épisode en question plutôt que de l'avoir trouvé ailleurs plus ou moins en l'état. À la lumière du caractère anthologique du manuscrit, je pencherais plutôt pour la retranscription d'un modèle plus ancien.

${ }^{18}$ En l'absence d'édition critique, une telle affirmation est naturellement assez téméraire. Mais elle reflète, je crois, l'état de nos connaissances actuelles.

${ }^{19}$ Les manuscrits de Guiron le Courtois parlent plus volontiers de Branor le Brun.
} 
doulx come une damoiselle, les yeulx roux et atrayans et si bien formé de tout le demourant du visage que c'estoit deduit que de le regarder. Les espaules eust belles et grandes par mesure, les bras eust longz et gros et les mains moult nervees et grandes a merveilles et moult avoit belles ongles. Le pis n'eust pas grandement, hors par le corps estoit assés gresle, les cuisses et les jambes eust plus belles que tous autre chevalier et trop seoit bien a cheval. Mais moult luy failloit bon cheval a le porter. Quant il estoit en estour, riens n'avoit duree devant luy et sambloit que luy tout $\left[3 \mathrm{v}^{\circ}\right]$ seul deust mener la chose a fin. Moult faisoit secretement ses chevaleries et trop estoit iré quant on le savoit. Autre mestier n'avoit que les armes, moult eust esté aymé des dames s'il y eust voulu entendre. Amoureux fut il une foys, mais non plus et moult ayma loyaument a celle foys la. Courtoys et gracieux estoit sur tous les chevaliers du monde et pour ce fut il surnommé ${ }^{20}$ le Courtoys. Moult mangeoit bien et peu buvoit. Travail enduroit plus voulentiers que nul autre et voulentiers oyoit le service divin. Trop aymoit ung chevalier quant il [l'] avoit a compaignon, moult fist de grans choses en son temps; mais plus eust fait, mais long temps demoura en prison par deux foys. Et portoit en ses armes ung escu d'or sans autre carge ${ }^{21}$ et le portoit pour l'amour de Galeoth le Brun, qui voulentiers portoit cest escu. Et ceulx qui ne congnoissoient Guiron le Courtois, l'appelloient le Chevalier a l'Escu d'Or. Tel estoit Guiron comme je vous ay compté. ${ }^{22}$

Cette notice, qui, après celle d'Arthur, ouvre donc la ronde des chevaliers, se caractérise non seulement par la volonté de décrire le protagoniste Guiron, mais aussi de bien l'enraciner dans son lignage qui est, après tout, un des lignages fondateurs de la chevalerie préarthurienne: sont mentionnés, Albrun et Dombart, Phébus le Fort, Hector, Branor et Galehaut, tous les trois nommés le Brun, dont on rappelle les débuts au Val des Bruns. L'auteur des armoriaux remonte donc aux origines des origines, au temps des géants. Or, le manuscrit Additional 36673 comporte lui aussi, comme d'autres recueils guironiens, une ouverture en clé «historique» qui propose un retour en arrière sur cette époque lointaine ${ }^{23}$. Mais il paraît être le seul à faire intervenir, et longuement, les deux ancêtres, Albrun et Dombart, qu'évoque la notice de l'armorial. En particulier, Albrun, père de Brun, occupe, dans le manuscrit londonien, un rôle important dans la guerre contre les géants, avec qui il finira par vivre en paix après avoir scellé une alliance politique entre hommes et géants ${ }^{24}$. Il est donc tentant de penser que l'auteur des notices des armoriaux arthuriens avait à sa disposition un manuscrit particulier de Guiron, proche du modèle de l'Additional $36673^{25}$.

\footnotetext{
${ }^{20}$ Le manuscrit fr. 12597 donne sur surnommé.

${ }^{21}$ Le manuscrit donne plutôt targe.

${ }^{22}$ Manuscrit de PARIS, BNF f. fr. 12597 , ff. $2^{\mathrm{v}}-3^{\mathrm{v}}$.

${ }^{23}$ On pense surtout à l'immense ensemble Paris, BNF, f. fr. 358-363. Cf. Lathuillère, Guiron le Courtois, op. cit., p. 70-74. Sur ce document, voir en dernier lieu B. Wahlen, « Du recueil à la compilation: le manuscrit de Guiron le Courtois, Paris, BNF fr. 358-363», Ateliers, 30, 2003, p. 89-100.

${ }^{24}$ Voir le résumé qu'en donne Lathuillère, Guiron le Courtois, op. cit., p. 482.

${ }^{25}$ Sur la base de détails du même ordre, j'ai jadis proposé une source assez spécifique aussi pour les personnages issus d'autres romans, qui pouvaient provenir d'un représentant post-
} 
Le reste de la généalogie proposée par l'armorial est également peu conforme à la doxa, il est vrai assez fluctuante, de l'ascendance guironienne, puisque la notice de l'armorial affirme que le héros fait partie du lignage des Bruns. Or, ce n'est pas ce que disent les rédactions les plus anciennes et les plus diffusées du Guiron. Selon elles, Guiron appartient bien à une lignée de première importance, mais qui part de Phébus le Fort et non des Bruns, et l'auteur des armoriaux réaffirme cette partie de la généalogie lorsqu'il rappelle l'épisode célèbre de la descente de Bréhus sans Pitié dans la cave familiale des ancêtres de Guiron ${ }^{26}$. C'est en effet le passage qui articule le plus clairement toutes ces données généalogiques par une expositio et une glose très élaborées que rendent possibles les découvertes de Bréhus: dans cette grotte, l'enfant terrible du royaume arthurien voit, touche et authentifie les tombeaux des aïeux du lignage qui y reposent et apprend leur histoire grâce à quelques briefs et aux explications fournies par le grand-père, un vieillard de plus de cent ans vivant reclus dans cette grotte en compagnie de son fils. Très clairement, tout ce passage a été composé pour glorifier les origines de Guiron par un long récit rétrospectif relatant l'histoire de Phébus le Fort, grand ancêtre du héros ${ }^{27}$. Aucune mention n'y est faite du lignage des Bruns qui ne paraît pas avoir joui d'une très grande réputation au moment où les versions les plus anciennes du Guiron ont été composées. Il faut attendre les compilations plus tardives, celle de Rusticien de Pise, les Prophécies Merlin et la grande fresque du fr. $358-363$ pour voir la branche de Guiron s'entremêler à celle des Bruns ${ }^{28}$.

vulgate de la matière arthurienne, comme le fr. 112. Comme les témoins français en sont peu nombreux et semblent se trouver surtout dans la bibliothèque des ducs de Milan et de Jacques d'Armagnac, on peut éventuellement circonscrire l'origine des armoriaux dans l'une ou l'autre de ces zones. Voir Clôtures du cycle arthurien, op. cit., p. 325-30. Il faudrait maintenant tenter de voir quel genre de manuscrit du Guiron se trouvait dans ces bibliothèques au $\mathrm{XV}^{\mathrm{e}}$ siècle. Il faudrait probablement faire une place de choix au manuscrit TORINO, Biblioteca Nazionale, L-I-9, sur lequel on verra F. Bogdanow, « Part III of the Turin Version of Guiron le Courtois : a hitherto unknown source of Ms. B.N. fr. $112 »$, Medieval Miscellany presented to Eugène Vinaver by Pupils, Colleagues and Friends, éd. A. H. Diverres, F. E. Sutcliffe \& F. Whitehead, Manchester-New York, Manchester University Press-Barnes \& Noble, 1965, p. 45-64.

${ }^{26}$ Le passage correspond aux $\S \S 110-15$ de l'analyse de Lathuillère, Guiron le Courtois, op. cit., p. 307-14. Le texte se lit dans l'édition d'A. Limentani, Dal «Roman de Palamedés » ai "Cantari di Febus-el-Forte», Bologna, Commissione per i testi di lingua, 1962 (Collezione di Opere inedite o rare 124), p. 40-188.

${ }^{27}$ Parmi les exploits figure en effet celui que rappelle la notice de l'armorial : avec trente-neuf compagnons, Guiron a vaincu cinq rois avant de mourir, à cause de son amour pour la princesse du Northumberland. L'histoire de Phébus se lit chez Limentani, Dal «Roman de Palamedés », op. cit., p. 78-184 ou, plus récemment, dans l'Anthologie de Guiron le Courtois. Édition et traduction d'épisodes choisis, vol. publié sous la direction de R. Trachsler, en collaboration avec S. Albert, M. Plaut et F. Plumet, Alessandria, Edizioni dell'Orso, 2004 (Gli Orsatti 22), p. 90-149 due aux soins de Sophie Albert.

${ }^{28}$ Pour une présentation fine et minutieuse du lignage des Bruns, voir la contribution de S. Albert, «Briser le fil - nouer la trame. Galehaut le Brun dans Guiron le Courtois », à paraître dans les Actes du colloque aixois de 2005, Façonner son personnage, éd. par C. Connochie- 
En l'occurrence, c'est la Compilation de Rusticien de Pise qui présente le plus de similitudes avec la notice de l'armorial: Hector le Brun a pour fils Galehaut le Brun et pour cousin Branor le Brun, lui-même oncle de Séguran le Brun. Comme Phébus le Fort est rattaché au même lignage, Guiron est automatiquement entraîné dans le mouvement de fusion ${ }^{29}$. Guiron devient lui aussi un Brun, comme l'affirme également sa notice dans l'armorial.

Par son écriture d'emblée fragmentaire ou, plus précisément, sélective, l'armorial n'est ni tenu de dessiner des arbres généalogiques complets ni de les expliciter pour chaque personnage. C'est ce qui apparait quand on examine les notices consacrées aux autres membres de la famille de Guiron, Séguran le Brun et Gallinan le Blanc, qui est le fils de Guiron. Ce n'est pas un «arbre», c'est à peine une branche ou une partie du tronc.

Dans cette construction, on comprend assez aisément la présence du seul rejeton guironien: Gallinan marque en quelque sorte l'aboutissement de la lignée dont les mythiques Albrun et Dombart, évoqués dans l'introduction de la notice du héros lui-même, constituaient le point de départ. Quant à Séguran, il s'impose en tant que représentant de la strate intermédiaire: il est un de ces Bruns de la «jeune » génération, il est là pour faire le lien entre les ancêtres et Gallinan. Séguran est encore gigantesque par sa taille, mais déjà absolument dissocié de tout ce que les personnages de taille surhumaine peuvent avoir d'inquiétant puisqu'il apparaît comme un tueur de monstre. On voit ainsi, en deux notices, tout le chemin parcouru par la chevalerie pré- ou protoarthurienne, qui passe du monde jeune des Bruns, Phébus et compagnie aux Gallinan, version dégénérée, dans tous les sens du terme, de la génération des pères. Si les premiers représentants du lignage des Bruns sont plus grands, plus forts et plus courageux que les hommes ordinaires, la notice de Gallinan sonne l'arrivée de la normalité. Les trois notices-Guiron, Séguran et Gallinan - condensent donc l'histoire du lignage entier.

Il reste à regarder un peu plus en détail les notices en question.

Cy devise de de Seguran le Brun

$\left[4 r^{\circ}\right]$ Seguran le Brun fut filz de Hector le Brun. Il estoit grant a merveilles que vous eussiés dit proprement que c'estoit ung geant. Le visage eust bel et grant et presque brun. Les cheveux eust plus noirs que d'autre couleur et de tout le demourant du corps estoit si bien formé per mesure qu'i n'y avoit riens que dire. Mais moult estoit doulx et paisible et n'aymoit guieres compaignie d'autre gens. De si demesuree force estoit que monstre ne geant n'avoit duree a luy. Il mangeoit si demesureement que dix hommes eussent esté bien repeuz de ce que failloit a celuy seul. Il portoit ung dragon en ses armes et c'estoit pour ce qu'il tua ung hydeux et terrible dragon quant il fut fait nouvel chevalier premierement. Et portoit en ses armes d'or a ung dragon de sable, armé, langué de sinople. ${ }^{30}$

Bourgne. J'emprunte à cette étude toute ma science et je remercie Sophie Albert de m'avoir fait parvenir son texte avant publication.

${ }^{29}$ Il Romanzo arturiano di Rustichello da Pisa, éd. F. Cigni, Pisa, Pacini Editore, 1994, § 15 et 38, p. 235 et 242 .

${ }^{30}$ Manuscrit de PARIS, BNF f. fr. 12597, fol. $4^{\mathrm{r}}$. 
Cette notice est intéressante, d'une part en raison de l'explication qu'elle fournit du meuble de l'écu et, de l'autre, du point de vue de la construction du lignage, car Séguran est introduit comme le fils d'Hector le Brun, alors que ce n'est pas normalement le cas : Séguran appartient bien au même lignage qu'Hector, mais apparaît en général décalé par rapport au héros : il est le neveu de Branor le Brun, qui est présenté comme un cousin d'Hector. Il est vrai que rien n'interdisait la création de la relation filiale entre Séguran et Hector puisque les textes étaient suffisamment souples dans l'exposition des liens généalogiques. Sans doute étaientils même trop souples, puisque la généalogie de Séguran est assez complexe, ce qui a précisément pu inciter l'auteur de l'armorial - ou son modèle - à en profiter à son tour $^{31}$.

En tout état de cause, il paraît s'agir d'un resserrement du lignage, où les héros descendent les uns des autres et les interstices sont progressivement éliminés ou comblés. Il n'était pas possible de laisser Séguran «sans père», il fallait lui en trouver un célèbre parmi les Bruns en place. Il est clair toutefois que l'absence, dans l'armorial, d'Hector le Brun et de Galehaut le Brun, qui forment le «vrai» couple père et fils dans toutes les traditions, a facilité la tâche de l'auteur des armoriaux qui a pu modifier cette descendance sans avoir à retoucher l'ensemble de l'arbre généalogique.

S'il n'est pas facile de déterminer l'existence d'un modèle pour la relation filiale entre Hector et Séguran, en revanche, il est plus aisé d'indiquer un passage parallèle pour l'épisode du combat contre le dragon puisqu'on retrouve ici assez précisément les données que présente un épisode conservé dans le manuscrit LONDON, British Library, Additional 36673. Séguran y vient à bout d'un dragon ailé rouge et noir mesurant trente-huit pieds de long, auquel il coupe d'abord les pattes de devant, puis la queue. Il fait ensuite soigneusement peindre sur ses armes l'image du monstre. ${ }^{32}$

Dans sa manière de reprendre cet exploit, on voit bien la façon de travailler de l'auteur de l'armorial. Il sait condenser les aventures, en garder l'essentiel, souvent sous forme d'écu ou de surnom - il faut se rappeler que Séguran est connu aussi sous le nom de Chevalier au Dragon - et "fonder» ainsi les deux. Surtout quand il s'agit de personnages secondaires sur lesquels on sait peu de choses, le portrait moral et la description physique, bref toutes les caractéristiques prêtées aux chevaliers, découlent simplement du nom et de l'écu : ici, on apprend que Séguran ressemble à un géant, tout en étant doulx et paisible, qu'il mange pour dix mais qu'il est un peu solitaire. Toutes ces précisions n'engagent à rien, mais contribuent à créer

\footnotetext{
${ }^{31}$ Il est vrai que Séguran est dit, à un certain moment, le fils de Brunor le Brun dans le manuscrit LONDON, British Library, Additional 36673, ce qui devrait interdire des modifications trop appuyées. Cf. Lathuillère, Guiron le Courtois, op. cit., § 259, p. 487. Pour d'autres traditions, voir l'index de l'ouvrage de Löseth, Le roman en prose de Tristan, op. cit., p. 536, pour les différents renvois. Voir en particulier ses observations p. 434-37. On revient vers la version du fr. 358. Je n'ai pas pu accéder à l'édition, citée par Sophie Albert, due aux soins de J. F. Levy, Livre de Meliadus: an Edition of the Arthurian Compilation of BNF fr. 340 Attributed to Rusticien de Pise, Berkeley, University of California, 2000, § 25183 et $§ 310-29$.

${ }^{32}$ Résumé par Lathuillère, Guiron le Courtois, op. cit., § 259, p. 488.
} 
un portrait assez cohérent, où l'auteur des armoriaux a su transformer en allusions ou en rappels laconiques les aventures et exploits narrés dans ses sources.

Le mécanisme est légèrement différent pour Gallinan, le fils de Guiron. D'une part, la biographie de Gallinan, dans Guiron le Courtois, comporte plusieurs épisodes plus ou moins développés dont il fallait tenir compte, et, surtout, ces épisodes sont loin d'être glorieux. Tous les défauts des chevaliers arthuriens étant systématiquement atténués dans l'armorial, l'auteur s'est donc trouvé devant la tâche délicate de décrire Gallinan sans le charger ${ }^{33}$.

Cy devise de Gallinant le Blanc, surnommé l'Orguilleux

Gallinant fut filz de Guiron le Courtoys et fut ung des bons chevaliers du monde de son corps. Mais si trescruel estoit qu'il $\left[4 \mathrm{v}^{\circ}\right]$ effaçoit la bonté de sa chevalerie. Il estoit grant et de belle taille. Les cheveulx roux et crespés et les yeuls vers et clers, mais fiert [!] regart avoit et despiteux. Belles espaules avoit et larges, bras et poingz eust long et gros et bien noez. Tout le demourant du corps estoit si bien fait qu'onques chevalier eust de son temps. Et estoit moult aisé dedans ses armes et mieulx chevauchoit que tout autre et trop bien ferant de lance et d'espee et bien le monstra apertemant. Sa bonne chevalerie tenoit il de son pere et la cruaulté qu'il avoit tenoit a cause de sa nourriture, car le compte fait mencion qu'il avoit esté nourry entre les plus cruelles gens du monde comme le livre de Guiron racompte. Ce Gallinant fu le darnier du lignaige des Bruns, et Palamidés le tua aprés qu'il eust abatus maintz chevaliers de la Table Ronde. Et portoit en ses armes d'or a ung dragon de gueulles, armé, langé de sable. Et tel estoit Gallinant comme je vous ay devisé, et ce fut la fin du lignaige des Bruns. ${ }^{34}$

Cette notice appelle plusieurs observations, la première concernant la préhistoire, qui, visiblement, est supposé connue : Guiron et Bloie, la future mère de Gallinan, sont emprisonnés traîtreusement par un chevalier nommé Gallinan. C'est dans les geôles du château de Gallinan que naît l'enfant, causant la mort de sa mère. Pour la grande beauté de l'enfant, le seigneur du château l'adopte et lui donne son nom. Malheureusement, il confie le nourrisson à sa soeur, plus cruelle encore que lui-même, dont le lait, selon la conception dominante au Moyen Âge, gâchera les bonnes teches des parents. C'est ainsi que s'expliquent les nombreux méfaits commis par le fils de Guiron qu'évoque aussi la notice de l'armorial. Sa mort, de la main de Palamède, est également conforme à la tradition, si bien que l'on a une petite notice biographique à la fois complète et représentative de l'image du personnage, même si elle atténue quelque peu les crimes de Gallinan ${ }^{35}$.

Le seul point qui pose véritablement problème est le surnom du personnage qui se trouve souvent dans les rubriques: Gallinan le Blanc est, dans la plupart des versions, le nom du père adoptif de l'enfant, le fils s'appelant Gallinan le Noir. Les

${ }^{33} \mathrm{La}$ tendance au blanchissement se vérifie, par exemple, pour des personnages aussi périlleux que Agravain, Mordret ou Gauvain. J'ai évoqué cet aspect dans Clôtures du cycle arthurien, op. cit., p. 340-47.

${ }^{34}$ Manuscrit de PARIS, BNF f. fr. 12597, ff. $4^{\mathrm{r}}-4^{\mathrm{v}}$

${ }^{35}$ Résumé par Lathuillère, Guiron le Courtois, op. cit., § 130, p. 335. Pour les allusions à sa mort, voir $\S 185$ et $\S 288$. 
manuscrits précisent même que «il fu apelés noirs pour ce que ses peres estoit

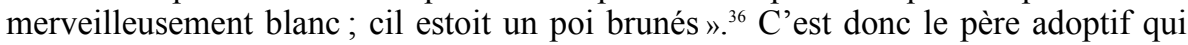
est clair et le fils qui est foncé. Le fils est nommé Gallinan le Blanc uniquement dans le fr. 112, qui entretient des relations étroites avec le manuscrit de Turin, vers lequel, décidément, tout converge ${ }^{37}$. Gallinan y est surnommé le Blanc et le père adoptif change de nom pour s'appeler Helin, allégeant ainsi le champ onomastique de son encombrante présence pour laisser la place à Guiron, le vrai père biologique.

Et c'est donc bien ainsi que s'achève l'histoire du lignage des Bruns dans les armoriaux : de Guiron lui-même, on passe, après un bref rappel des origines des Bruns, à Séguran, puis à Gallinan, dont la devise est Filz Guiron, comme s'il fallait afficher dans les armes ce que le caractère et les faits ne permettaient pas de deviner ${ }^{38}$. Guiron, dans l'armorial, est bien le point de repère du lignage entier.

En France, la littérature arthurienne, au $\mathrm{XV}^{\mathrm{e}}$ siècle, est plus marquée du sceau de la recréation que de la création. On met en prose ce qui existe déjà, on le dérime. C'est indéniablement l'époque des Erec, Cligés et Floriant en prose. La seule autre option est celle de la somme, où l'on compile ce qui était épars, où l'on coupe, rajoute, rassemble, soigne la forme, trafique le détail, mais où l'on n'» invente" rien. C'est le règne des assemblages, des sommes homogènes, comme la grande saga arthurienne que contient le fr. 112 transcrit par Michel Gonneau et l'immense ensemble PARIS, BNF, f. fr. 358-363, ou des montages plus éclectiques, comme le Tristan de Pierre Sala.

Dans ce contexte, les armoriaux arthuriens sont à la fois conformes et réfractaires à la tradition dominante. Conformes parce que, en apparence, ils n'inventent rien, ils se contentent de «recycler»; et c'est précisément pour cela qu'on peut les considérer aussi comme réfractaires à la tradition dominante de la fin du Moyen Âge. Les armoriaux brisent le flux d'une narration continue et substituent à l'esthétique héritée de la littérature arthurienne une forme nouvelle qui n'est ni celle de la somme ni celle de la compilation telles qu'elles existent dans la tradition antérieure: plus qu'un cycle, les armoriaux composent un cercle arthurien. La matière y est disposée de façon différente, pour créer un livre où il ne se passe presque rien, au moment précis où la littérature narrative joue plutôt la carte de la surenchère et de l'action tous azimuts. Avec le cadre de la Pentecôte du Graal, qui fige en un lieu et un moment uniques cent cinquante chevaliers de la Table Ronde, l'armorial est un still life et non un roman puissance cent cinquante.

La présence de chevaliers plus anciens d'une génération brise quelque peu ce cadre. Elle constitue une première strate, élaborée sur le modèle des chevaliers de la Table Ronde, qui paraît bien répondre à une des grandes préoccupations en matière de littérature narrative à la fin du Moyen Âge : la tendance à la complétude. Après l'apparition de leurs romans à eux, la génération de Guiron, de Meliadus, mais aussi

${ }^{36}$ Texte du manuscrit fr. 350, cité d'après Lathuillère, Guiron le Courtois, op. cit., § 120, p. 322.

${ }^{37}$ Lathuillère, Guiron le Courtois, op. cit., § 288, p. 525. Voir, sur le manuscrit de Turin, les observations de Fanni Bodganow, citées supra.

${ }^{38}$ Pour la devise, voir Pastoureau, Armorial des Chevaliers de la Table Ronde, op. cit., p. 56. 
de Balaain et de Pellinor, ne pouvait manquer à l'appel. Il a fallu leur inventer des physiques, des habitudes vestimentaires et gastronomiques, ainsi que des parents, comme pour leurs fils. Pour ce faire, l'auteur s'est servi de son imagination, mais guidée par ses lectures. Ces armoriaux constituent donc à double titre une trace de la réception de la littérature arthurienne à la fin du Moyen Âge : d'une part, parce que ces descriptions montrent comment on se figurait les chevaliers de la Table Ronde au XV siècle, et, d'autre part, parce qu'on saisit, à travers ces notices, des traditions textuelles dont nous avons certes connaissance par ailleurs, mais dont nous avons peu d'autres témoins. Il faut donc lire et relire ces descriptions, qui sous leur dehors répétitif révèlent à leur manière des aspects essentiels de la littérature arthurienne de la fin du Moyen Âge.

Richard Trachsler Université de Paris IV-Sorbonne / Institut Universitaire de France 